\title{
Helium-3 purification system and acoustic cavity for concentration determination
}

\section{Carlos Banuelos Perez, Wenguang Jiang \\ University of Florida}

Faculty mentor: Yoonseok Lee, Department of Physics

\begin{abstract}
This paper discusses a filtering method of using differential adsorption to purify helium-3 $\left({ }^{3} \mathrm{He}\right)$ from a helium-4 $\left({ }^{4} \mathrm{He}\right)$ impurity. In addition, the paper discusses the precision of an acoustic cavity in determining the concentration of a mixture through measuring the speed of sound for a given sample. Data analysis will include measurements at room temperature in air at $1 \mathrm{~atm},{ }^{4} \mathrm{He}$ at $\approx 1.2 \times 10^{5} \mathrm{~Pa}$ and $\approx$ $1.6 \times 10^{5} \mathrm{~Pa}$, and Nitrogen at $\approx 1.0 \times 10^{5} \mathrm{~Pa}$ resulting in a percentage error that ranges from $1.03 \%$ $2.55 \%$.

Keywords: helium-3, purification, acoustic cavity
\end{abstract}

\section{Introduction}

Helium-3 is the lighter and extremely rare isotope. Considering that the total amount of helium composes only 5.4 ppmv of the earth atmosphere, and helium-3 is a product of tritium decay collected from nuclear weapons, it is not surprising to know that helium-3 is arguably the most expensive element by weight (about \$1,000 per STP liter). Eighty-five percent of the helium-3 is used for neutron detectors for security screening, which is the highest priority, and about $3 \%$ is used for basic science research (Feder, 2009).

Interestingly, helium-3 itself is a main topic of research in low-temperature physics because of its unique physical properties in the form of liquid at extremely low temperature where quantum mechanics is relevant. Two isotopes of helium are the only elements that cannot be solidified down to absolute zero below melting pressure ( 25 bar for helium-4 and 34 bar for helium-3) (Dobbs, 1994). Unlike helium-4, the helium-3 atom carries a half-integer spin (nuclear spin $1 / 2$, electron spin 0) and becomes a composite fermionic quantum particle below $100 \mathrm{mK}$ (Dobbs, 1994). Liquid helium-3 exhibits a remarkable quantum phenomenon called superfluidity below $2 \mathrm{mK}$ which is in close analogy with superconductivity occurring in charged fermionic systems (Dobbs, 1994). 
In low-temperature laboratories using helium-3 as a sample and also a refrigerant, it is crucial to establish an infrastructure to minimize the loss of this valuable resource. In many occasions, intentionally and unintentionally, the pure helium-3 gas can be polluted by impurities. Among those impurities, helium-4 impurity in helium-3 is the hardest to remove due to the close boiling points: 3.2 K for helium-3 and 4.2 K for helium-4 (Dobbs, 1994). Conventional systems, separating helium-3 and helium-4, use rectification and fractional distillation methods which are time-consuming, require expensive equipments and are sensitive to pressure and temperature changes (Dmitriev et al, 2004). Instead, the system for helium extraction and purification project (SHeEP) utilizes the difference in adsorption energy by activated charcoal between the helium isotopes. SHeEP utilizes a two-stage purification process which first eliminates nonhelium contamination by forcing them through a nitrogen trap then eliminates helium- 4 isotope through a helium cold trap. SHeEP also requires a method to monitor the process of purification. At a given temperature, the usage of an acoustic resonance cavity will obtain the concentration of the mixture through measuring the speed of sound in the sample (Lin et al, 2013).

\section{A System for Helium Extraction and Purificaiton (SHeEP)}

As shown in Figure 1, SHeEP consists of the $\mathrm{N}_{2}$ cold trap, the cryo pump, multiple pressure gauges, a chromatographic column filled with activated charcoal $\left({ }^{4} \mathrm{He}\right.$ trap), and the acoustic cavity for purity monitor which are also shown in Figure 2 including three containers (for storing gases at different concentration levels after purification). Gas flow is driven by the Agilent IDP-3 scroll pump through $1 / 4$ " copper tubing and containers. The $\mathrm{N}_{2}$ cold trap, the cryo pump, and the acoustic cavity are connected to the main gas handling system (GHS) through $1 / 16$ " stainless steel tubing. Table 1 provides further information of the dimensions of the traps and cryo pump including the amount of charcoal in each.

An impure sample consisting of the Helium isotopes with air (held in container 3), will first need to be routed through the $\mathrm{N}_{2}$ cold trap. The $\mathrm{N}_{2}$ cold trap, similar to the Helium trap, holds activated charcoal to cleanse the sample from air molecules by setting the cold trap in liquid nitrogen. This condenses and traps air molecules, where the charcoal provides additional surface area to trap the condensed air molecules, allowing the Helium isotopes in gas form to escape. To separate the Helium isotopes, a similar process is used except the activated charcoal filter is

engulfed in liquid Helium which traps the heavier ${ }^{4} \mathrm{He}$ and allows ${ }^{3} \mathrm{He}$ to escape. The traps are 
cleaned, and the process is repeated again. To ensure purity, a sample is routed to the acoustic cavity and the concentration is measured from the sound velocity measurement.

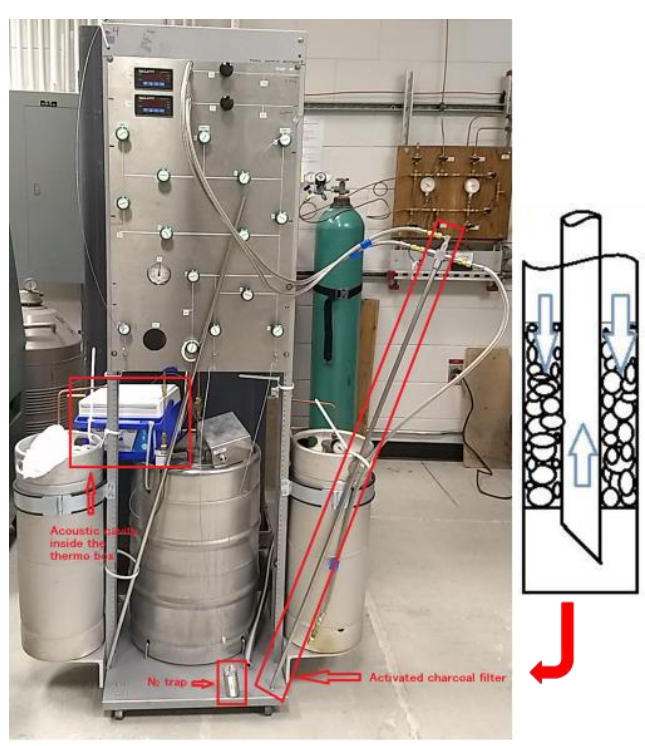

(a) (b)

Figure 1. (a) System for Helium extraction purification. (b) Schematic diagram of the He trap.

A cryo pump is installed to facilitate gas handling. It is also a column filled with activated charcoal which draws gas in at a lower temperature and exhales at a higher temperature. It significantly expands the pressure range available to us.

Figure 2 is a schematic drawing of the panel used in the SHeEP. The panel shown consists of $\mathrm{G} 1$ and $\mathrm{G} 2$ which measures the pressure out of the pump and the pressure of the acoustic cavity, respectively. Figure 2 also shows TG1 and TG2 which measures the pressures on the front and back ends of the ${ }^{4} \mathrm{He}$ trap, respectively. During purification of gas from container 3 to container 2, valves C, G, N, O, T1, T2, Q, F, D, B are open, and gas flows through these valves in this given order, where the flow rate is controlled by valves T1 and T2. One could also cycle the gas in the SHeEP indefinitely by having the valves D, E, G, N, O, T1, T2, Q, F open, and pump gas through these valves in the given order. Purifying gas from container 2 to container 1 can be done in a similar fashion. The volumes directly connected to the pump, between valves $\mathrm{T} 2$ and $\mathrm{Q}$, are sealed by $\mathrm{KF}(\mathrm{QF})$ flanges, and cannot be pressurized beyond $1 \mathrm{~atm}$. 


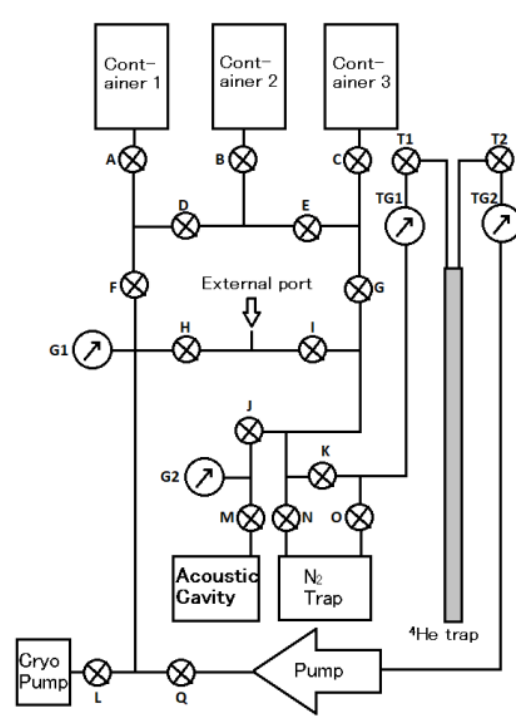

Figure 2. Schematic diagram of SHeEP. The gray bar on the right side represents the ${ }^{4} \mathrm{He}$ trap.

Table 1. Dimensions and Charcoal Amount

\begin{tabular}{lccc}
\hline $\begin{array}{l}\text { Filtering } \\
\text { Apparatus }\end{array}$ & \multicolumn{3}{c}{ Corresponding Dimensions } \\
\hline & $\underline{\text { Length }}$ & $\underline{\text { Diameter }}$ & $\underline{\text { Charcoal }}$ \\
& $\underline{\text { in. })}$ & $\underline{\text { (in.) }}$ & $\underline{\text { mass }(\mathrm{g})}$ \\
${ }^{4} \mathrm{He}$ Column & 15.63 & 0.5 & 19.12 \\
$\mathrm{~N}_{2}$ Trap & 3.0 & 1.5 & 31.22 \\
Cryo Pump & 5.0 & 0.5 & 5.51 \\
\hline
\end{tabular}

Note. The surface area of the charcoal used is $200 \mathrm{~m}^{2} / \mathrm{g}$.

\section{Design and Performance of Acousitic Cavity}

The quality $(\mathrm{Q})$ factor of a cylindrical cavity are limited by four factors:

(1) Viscous damping of the motion of the gas in contact with the cylinder walls.

(2) Heat loss at walls due to heat conduction during adiabatic compression-decompression cycle.

(3) Tranducer loss during the measurement.

(4) Losses through the openings in the cavity. For example, a filling hole on the wall of the cavity will allow gas flow (into and out of the cavity) due to pressure change caused by 
acoustic waves. This gas flow is not isentropic and dissipates energy through volume viscosity (or second viscosity).

The influence of item (4) can be minimized by placing the filling hole on the same spot as the pressure node at the middle on the cavity side wall.

The contributions of (1) and (2) to the total Q factor can be estimated as

$$
\frac{1}{\mathrm{Q}_{t o t}}=\frac{\delta_{\eta}}{a}+\frac{\delta_{\kappa}}{L}
$$

where $\delta_{\eta}=\sqrt{\frac{2 \eta}{\rho \omega}}$ and $\delta_{\kappa}=\sqrt{\frac{2 \kappa}{c_{p} \rho \omega}}$ are the viscous and thermal penetration depth respectively (Kinsler et al, 1999). Here, $\eta$ is the gas's coefficient of shear viscosity, $\kappa$ is its thermal conductivity, and $c_{p}$ is the specific heat at constant pressure. From this, we determine the optimal radius to length ratio for the cavity in order to achieve the highest $\mathrm{Q}$ factor. The optimal ratio is

$$
\frac{L}{2 a}=\sqrt{\frac{\kappa}{\eta c_{p}}}=1.11
$$

for $\kappa \approx 70 \times 10^{-3} \frac{\mathrm{W}}{\mathrm{mK}}, \eta \approx 0.82 \times 10^{-5} \mathrm{~Pa} \cdot s, c_{p}=6928 \frac{\mathrm{J}}{\mathrm{K} \cdot \mathrm{kg}}$. Furthermore, for $2 a=$ $5.94614 \times 10^{-2} \mathrm{~m}, L=5.4864 \times 10^{-2} \mathrm{~m}, \delta_{\eta}=2.35 \times 10^{-5} \mathrm{~m}$ and $\delta_{\kappa}=2.61 \times 10^{-5} \mathrm{~m}$ gives $\mathrm{Q}_{\mathrm{tot}} \approx 300$.

Now, we need to consider an estimation of the sensitivity of the cavity when used as a tool for measuring the concentration of a binary mixture of gases. Specifically, we discuss pure ${ }^{3} \mathrm{He}$ gas contaminated by a small amount of ${ }^{4} \mathrm{He}$. The concentration of ${ }^{3} \mathrm{He}$ is $1-\mathrm{x}$, and $\mathrm{x}$ for ${ }^{4} \mathrm{He}$. Their properties will be subscripted by 3 and 4 respectively. The speed of sound in an ideal gas is (Blundell \& Blundell, 2010).

$$
c^{2}(x)=\gamma(x)\left(\frac{k_{B} T}{m(x)}\right),
$$

where $\gamma_{x}=\gamma_{3}(1-x)+\gamma_{4} x, \gamma=c_{p} / c_{v}$ is the ratio of specific heats at constant pressure and volume, and $m(x)=m_{3}(1-x)+m_{4} x$ is the average molecular mass of the gas (Polturak et al, 1986; Kagiwada \& Rudnick, 1970).

The speed of sound for an ideal gas does not depend on the density of the gas, but only it's compositon. ${ }^{4} \mathrm{He} /{ }^{3} \mathrm{He}$ mixture at room temperature and pressure of $\approx 1.0 \times 10^{5} \mathrm{~Pa}$ can be treated as an ideal gas.

The measurement is done by comparing the speed of sound in the mixture to that in the pure ${ }^{3} \mathrm{He}$ (Polturak et al, 1986). 


$$
\left(\frac{\mathrm{c}(\mathrm{x})}{\mathrm{c}(0)}\right)^{2}=\frac{\gamma(x)}{\gamma(0)} \frac{m(0)}{m(x)}=\left[1+\left(\frac{\gamma_{4}-\gamma_{3}}{\gamma_{3}}\right) x\right] /\left[1+\left(\frac{m_{4}-m_{3}}{m_{3}}\right) x\right] .
$$

If $\mathrm{x}<<1$, the variation of $c(x), \delta c(x)$, can be expressed as a function of the variation of $x, \delta x$ :

$$
\frac{\delta c(x)}{c(0)}=\frac{\delta x}{2}\left[\frac{\gamma_{4}-\gamma_{3}}{\gamma_{3}}-\frac{m_{4}-m_{3}}{m_{3}}\right] .
$$

If some $m \gg m_{3}$ is present, the term after the minus sign will become significantly large. This means that outgasing or any other source of gaseous contamination should be avoided, possibly through long pumping time and cold traps. Continuing to estimate $\delta x$, plugging in the Boltzmann constant, the atomic mass and the speed of sound of ${ }^{3} \mathrm{He}$ respectively, gives

$$
\frac{\delta c(x)}{c(0)}=\frac{\delta x}{2}\left(-\frac{1}{3}\right)
$$

from which one can obtain the expression for

$$
\delta x=-6 \frac{\delta c(x)}{c(0)}=-6 \frac{\delta f_{0}}{f_{0}} .
$$

Our routine fitting program for Lorentzian peaks consistently produces $\frac{\delta f_{0}}{d}$ to have the order of magnitude of $\sim 0.01$. Plugging this into Eq.(7), we arrive at

$$
|\delta x|=6 \frac{\delta f_{0}}{d} \frac{d}{f_{0}}=6 \frac{\delta f_{0}}{d} \frac{1}{Q_{t o t}}=0.04 \% .
$$

Figure 3 and Figure 4 show a pictorial and schematic description of the acousitic cavity, respectively. The cavity is mirror symmetrical with a transducer on each end of it. The transducer is a mylar film (thickness $=30 \mu \mathrm{m}, \mathrm{ID}=2.12 \mathrm{in}$.) coated with gold on the side that faces the interior of the cavity. Figure 6 shows the film attached to a brass cap which houses a cylindrical copper electrode shown in Figure 5. The film is mechanically attached to the cap by stycast 1266 epoxy and electrically connected by E4110 silver epoxy, while the copper electrode is attached to but electrically insulated from the cap by $2850 \mathrm{FT}$ epoxy. The mylar film is parallel to the flat sides of the copper electrode, forming a capacitor with a 0.03 in. gap size. An estimated distortion of the film at $100 \mathrm{~V}$ DC bias is $120 \mu \mathrm{m}$. 


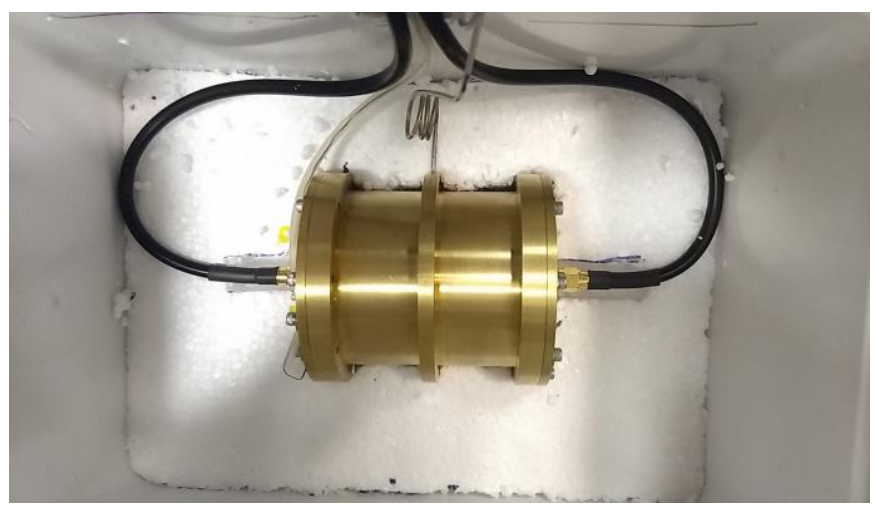

Figure 3. A top view of the acoustic cavity secured in a piece of Styrofoam. The top is covered by a separate piece of Styrofoam, which is removed here for the camera.

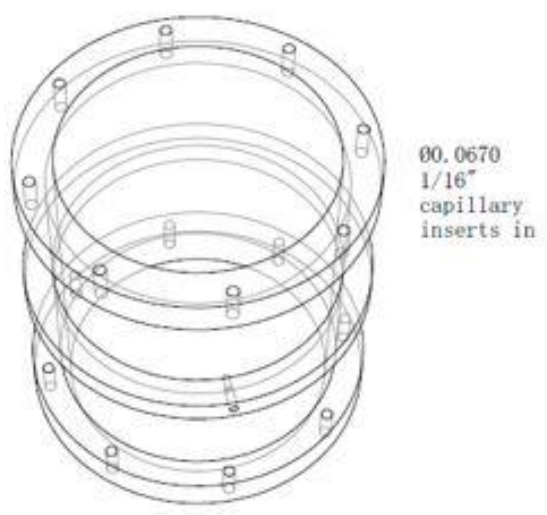

(a)

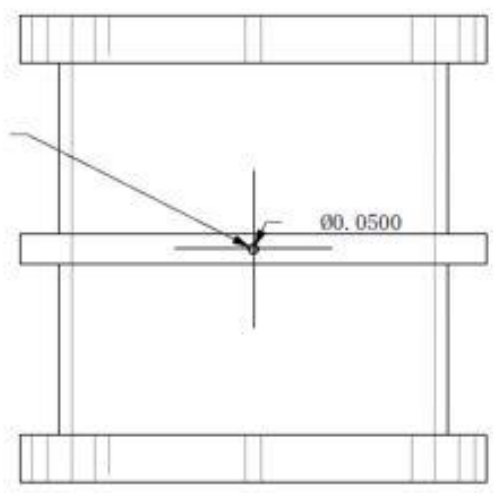

(b)

Figure 4. (a) Top view diagram of the acoustic cavity. (b) Side view diagram of the acoustic cavity.

The functionality of the transducers are to convert energy from one form to another, in this case electrical to mechanical and vice versa. Each is attached to the cavity body and used as the actuator and the detector. The temperature of the acoustic cavity is stabilized by holding the acoustic cavity in a thermo box filled with pieces of styrofoam. A resistance thermometer, Cernox CX-1080-CU-HT-20L, measures the temperature in the acoustic cavity. The pressure is measured by a Setra 206 pressure gauge (G2 in Figure 2) on the filling line. 


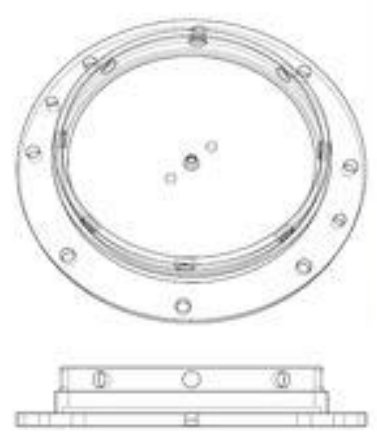

(a)

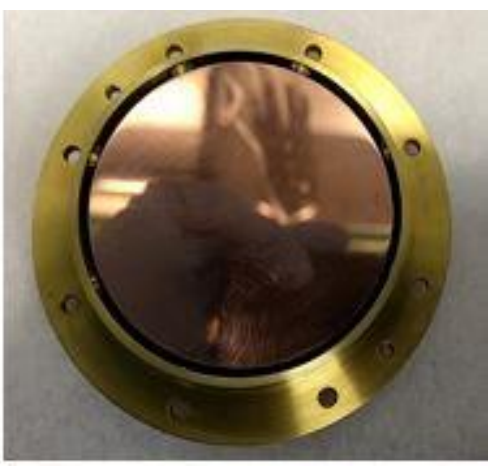

(b)

Figure 5. (a) Diagram model of transducer cap. (b) Inside view of the cap. The round center piece is the cylindrical copper electrode.

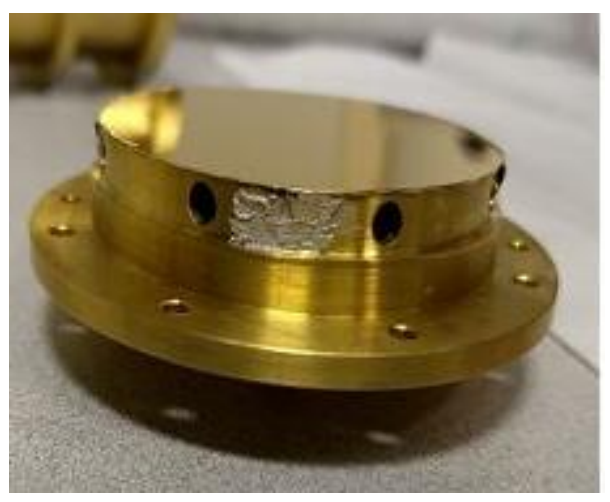

Figure 6. The mylar film already stretched and attached to the cap. This visible side is coated by gold. Some silver epoxy can be seen that serves as a electrical connection.

\section{Measurements of the Acousitic Cavity}

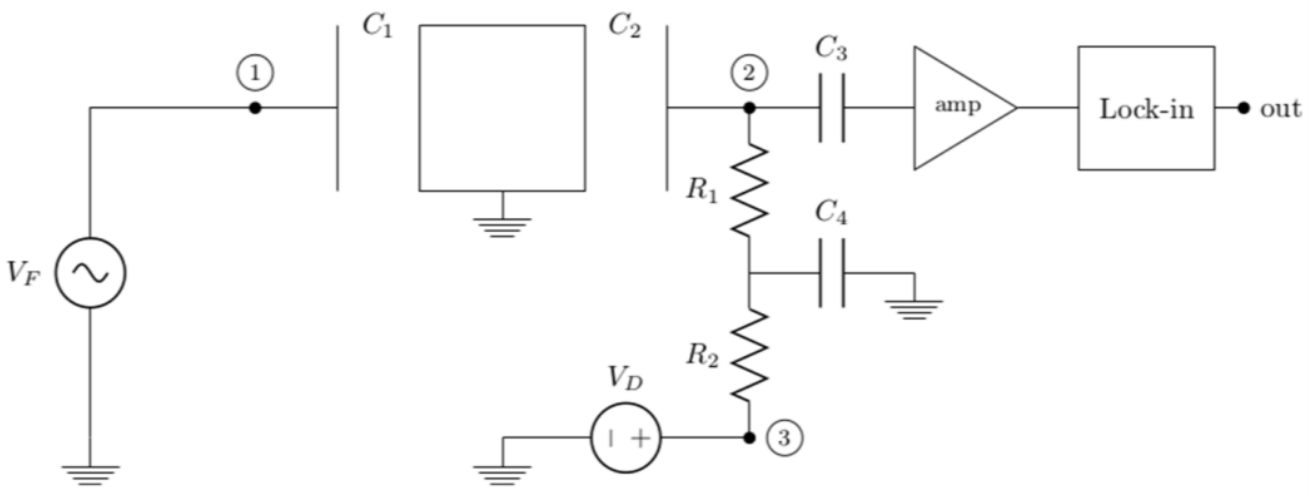

Figure 7. Schematic diagram of the circuitry of the acoustic cavity. 
Figure 7 is a schematic drawing of the circuit which is driven by a maximum AC voltage of $V_{F}=50 \mathrm{~V}_{\mathrm{pp}}$. Where $V_{F}$ is the voltage applied, the actuator is driven by $F_{a}=1 / 2 C\left(V_{F}^{2}+\right.$ $\left.V_{F}^{2} \sin (2 \omega t)\right)$, causing the film at capacitor $1\left(\mathrm{C}_{1}\right)$ to oscillate at $2 \omega$. Sound across the cavity body (the square between $\mathrm{C}_{1}$ and $\mathrm{C}_{2}$ ) now oscillates at $2 \omega$. Continuing, the film at capacitor 2 $\left(\mathrm{C}_{2}\right)$ now oscillates at $2 \omega . \mathrm{C}_{1}$ and $\mathrm{C}_{2}$ are calculated to be $\approx 30 \mathrm{pf}$. The detector is biased by a DC voltage $\left(50 \mathrm{~V}_{\mathrm{DC}}\right)$ to read the charge of $\mathrm{C}_{2}$, then the signal is translated and amplified in terms of voltage by the pre-amplifier and the lock-in amplifier. This voltage output is graphed against frequency to find the speed of sound through the sample.

\section{Results}

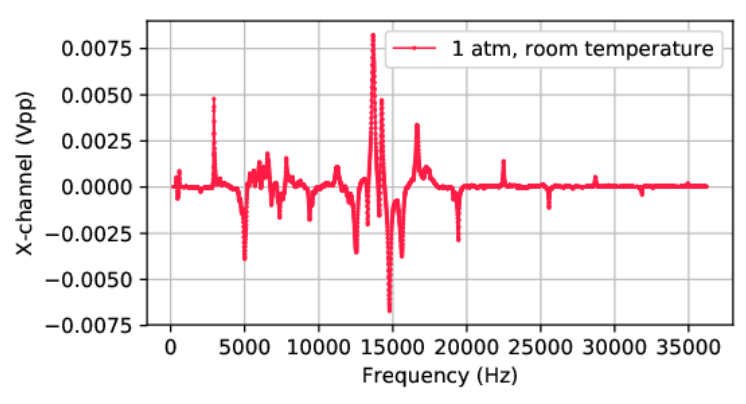

(a)

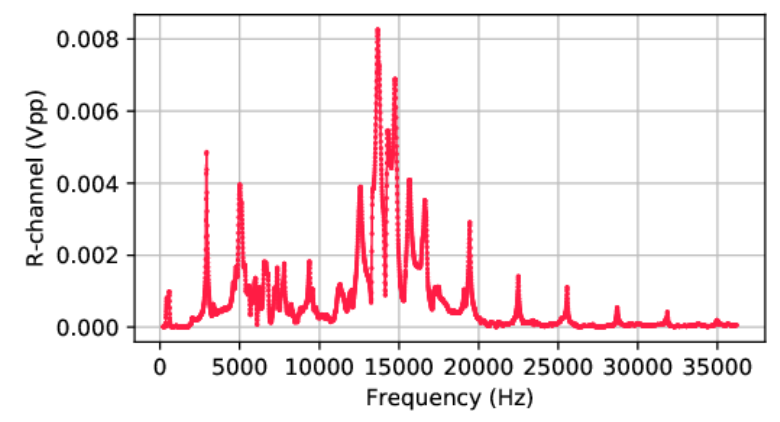

(c)

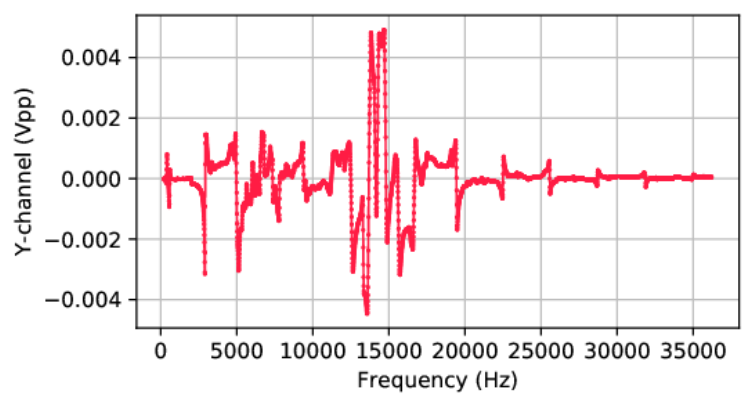

(b)

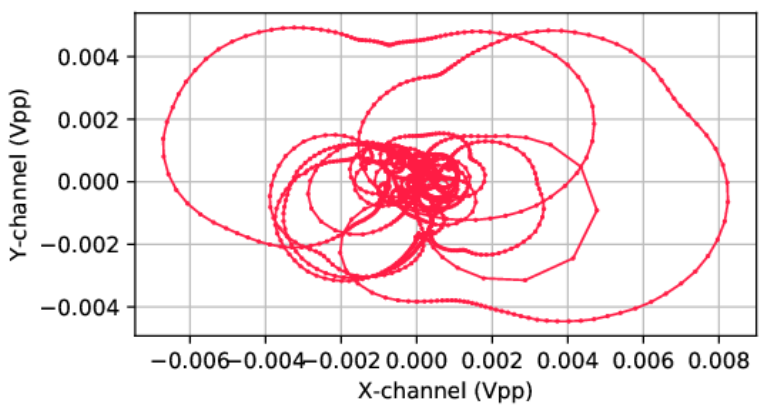

(d)

Figure 8. All plots were taken in air at room temperature. (a) The x-channel is the quadrature signal. (b) The ychannel is the in-phase signal. (c) The r-channel is the amplitude of the signal. (d) The Johnson-Nyquist plot.

Figure 8 are the results from a wide range frequency sweep of the acoustic cavity. Most of the features below about $18000 \mathrm{~Hz}$ are membrane circular vibrational modes. These features are so rich that they overwhelm the cavity gas resonance modes. Therefore, data analysis is only carried out in the high frequency range, where the cavity modes stand out of the severely damped membrane modes. A more refined frequency sweep is done for each peak, whose resonance 
frequency is fit to extract the width and resonance frequency. An example fitting for the peak at about $28700 \mathrm{~Hz}$ is shown in Figure 9 which is conducted on a handpicked frequency range from $28550 \mathrm{~Hz}$ to $28900 \mathrm{~Hz}$. The fitted resonance frequency $\mathrm{f}_{0}$ is $=28698.42 \mathrm{~Hz}$, the width $\mathrm{d}$ is $=$ $72.47 \mathrm{~Hz}(\mathrm{Q}=396)$.

Table 2 lists resonance peaks, width and quality factor that is assigned an integer $\mathrm{n}$, representing the number of half wavelengths of the resonating sound waves. The quality factor is

$$
Q=f_{0} / d \text {. }
$$

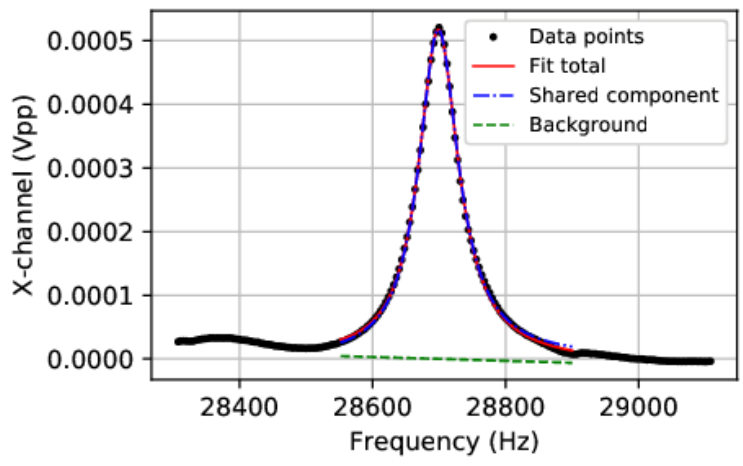

(a)

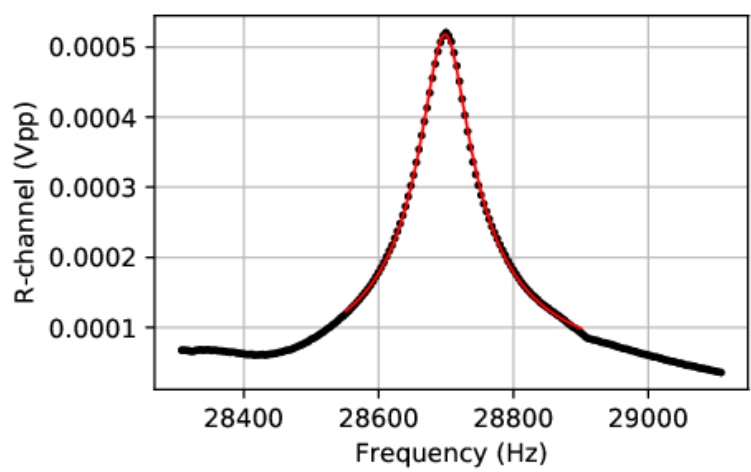

(c)

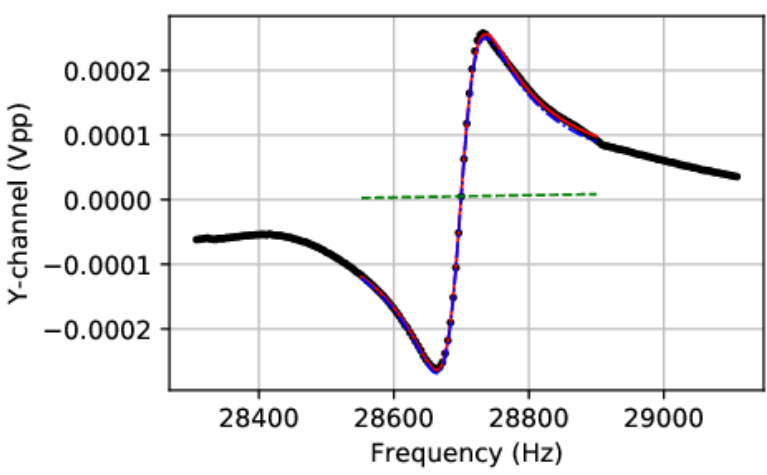

(b)

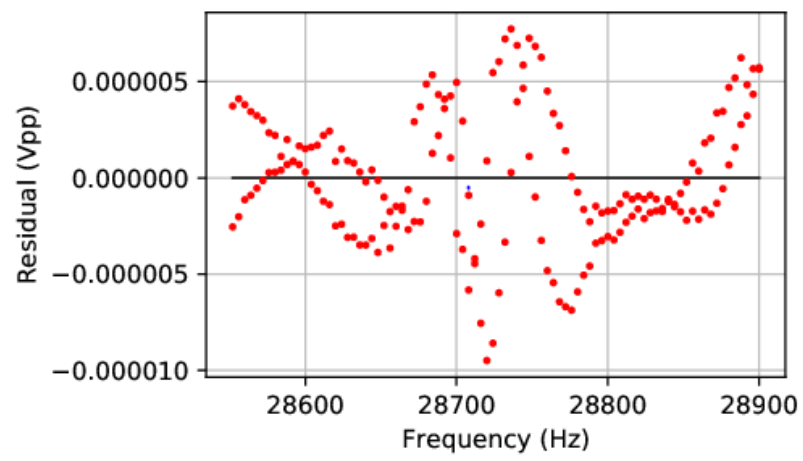

(d)

Figure 9. All plots were taken in air at room temperature. The fit (red solid line) includes a Lorentzian peak (blue dash-dot lines) on top of a background (green dashed lines). (a) The x-channel vs frequency with fit. (b) The ychannel vs. frequency with fit. (c) The r-channel vs. frequency with fit. (d) The difference between the fitted curve and the actual data. 
Table 1. Fitting result of all five higher frequency in air and room temperature.

\begin{tabular}{lccc}
\hline $\begin{array}{l}\text { Frequency } \\
\text { integer }\end{array}$ & \multicolumn{3}{c}{ Corresponding Values } \\
\hline$n$ & $f_{0}(\mathrm{~Hz})$ & $d(\mathrm{~Hz})$ & $Q$ \\
6 & 19451.79 & 95.89 & 202.9 \\
7 & 22498.44 & 72.81 & 309.0 \\
8 & 25562.98 & 55.78 & 458.3 \\
9 & 28698.42 & 72.47 & 396.0 \\
10 & 31857.26 & 38.52 & 826.9 \\
\hline
\end{tabular}

At resonance, the length of the cavity, $\mathrm{L}$, is a half-integer multiple of the wavelength:

$$
L=\frac{n \lambda}{2}=\frac{n c}{2} \mathrm{f}_{0}{ }^{-1}
$$

which shows that the resonace frequency $\left(\mathrm{f}_{0}\right)$ is linearly dependent on $\mathrm{n}$ :

$$
\mathrm{f}_{0}=\frac{n c}{2 L} \equiv \Lambda n
$$

where $\lambda$ is the wavelength of the sound wave, and $\mathrm{c}$ is the speed of sound. The slope can then be used to calculate

$$
c=2 L \Lambda \text {. }
$$

A linear regression is done on the $\mathrm{f}_{0}$ and $\mathrm{n}$ data, and the result is depicted in Figure 10. The fitted slope is $\Lambda=3101.09 \mathrm{~Hz}$, with a standard deviation of $0.10 \mathrm{~Hz}$. Oddly enough, the fit also gives a non-zero intercept that equals $805.06 \mathrm{~Hz}$ with a standard deviation of $0.87 \mathrm{~Hz}$. According to the design, the distance between the two transducer membranes is $\mathrm{L}=2.160$ in. $=5.4864 \times$ $10^{-2} \mathrm{~m}$. Using this, $c=2 \times 5.49 \times 10^{-2} \times 3101.09=340.276 \mathrm{~m} / \mathrm{s}$.

Similar measurements have been performed in ${ }^{4} \mathrm{He}$. At $1.2 \times 10^{5} \mathrm{~Pa}$, room temperature (the measured pressure is $119934 \mathrm{~Pa}$ before, and $119506 \mathrm{~Pa}$ after the sweep; the temperature is 297.4 $\mathrm{K}$ before and $297.4 \mathrm{~K}$ after the sweep), and $1.6 \times 10^{5} \mathrm{~Pa}$, room temperature (the measured pressure is $159981 \mathrm{~Pa}$ before, and $160336 \mathrm{~Pa}$ after the sweep; the temperature is $295.8 \mathrm{~K}$ before and $295.2 \mathrm{~K}$ after the sweep). Three peaks are identified as acoustic wave resonance modes. Figure 11 plots the two measurements swept in ${ }^{4} \mathrm{He}$ under the aforementioned conditions. A zoomed in version is seen from Figure 12, focusing within the frequency range from 25,400 to $47,000 \mathrm{~Hz}$. 


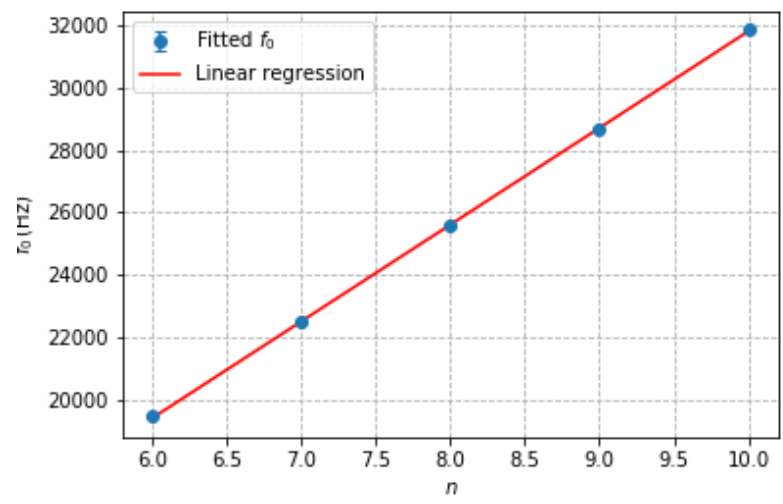

Figure 10. $\mathrm{f}_{0}(\mathrm{~Hz})$ vs. $\mathrm{n}$ plot. The dots are $\mathrm{f}_{0}$ obtained from the Lorentzian fits, the line is generated by linear regression.

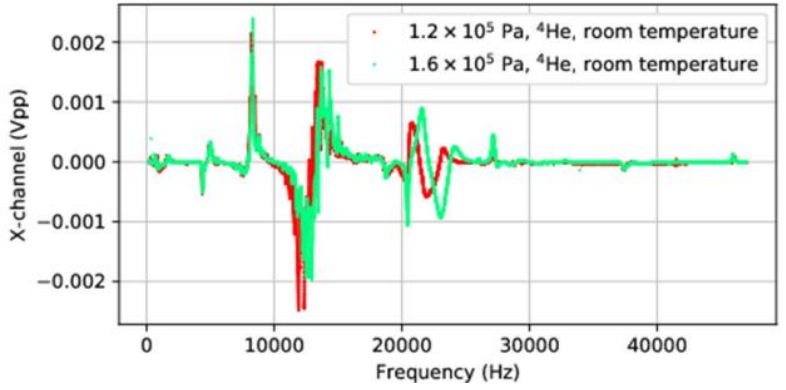

(a)

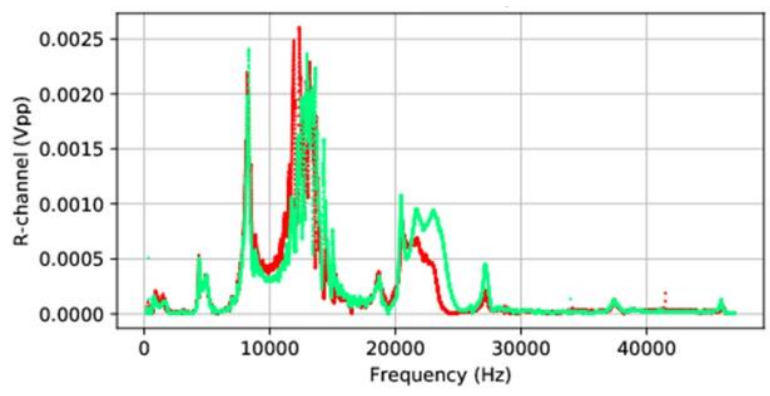

(c)

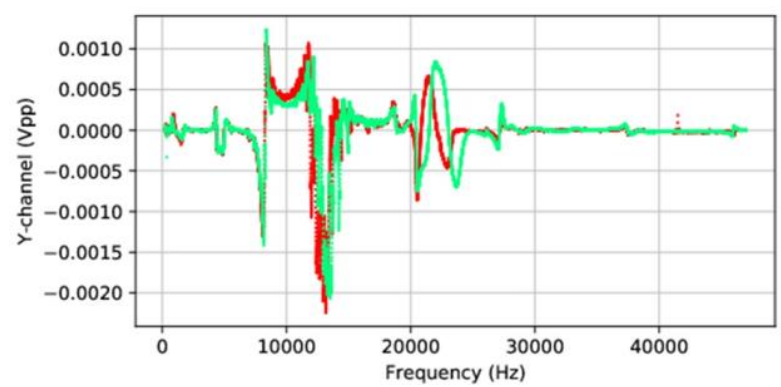

(b)

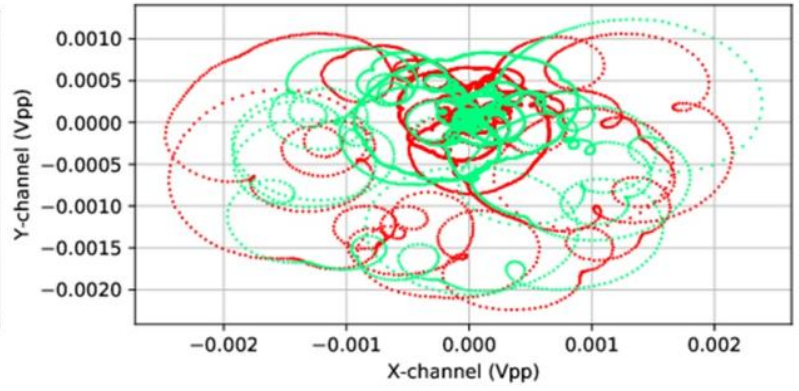

(d)

Figure 11. (a) The $x$-channel is the quadrature signal. (b) The $y$-channel is the in-phase signal. (c) The r-channel is the amplitude of the signal. (d) The Johnson-Nyquist plot. 


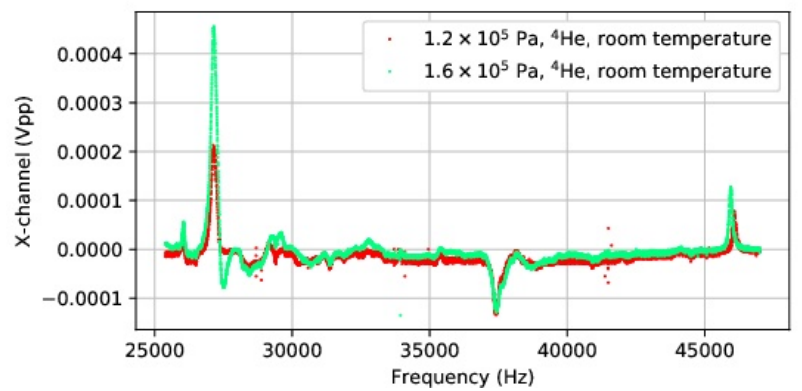

(a)

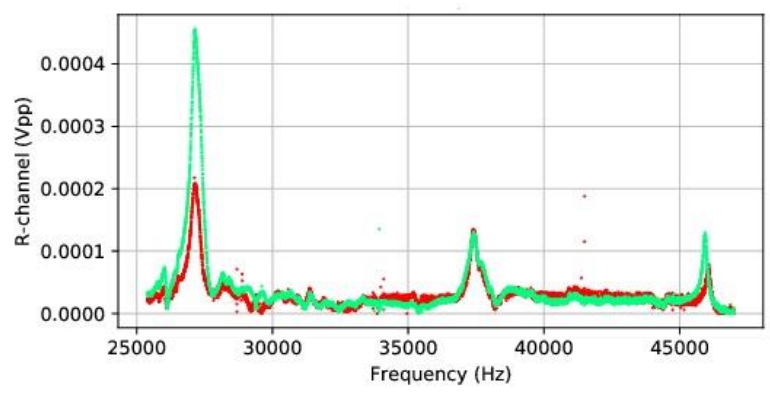

(b)

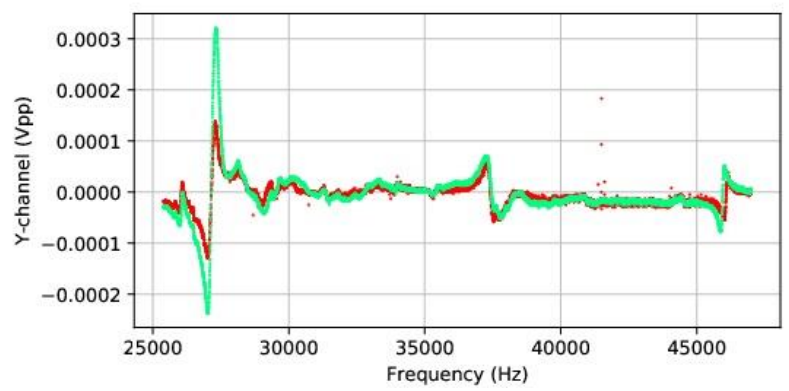

(b)

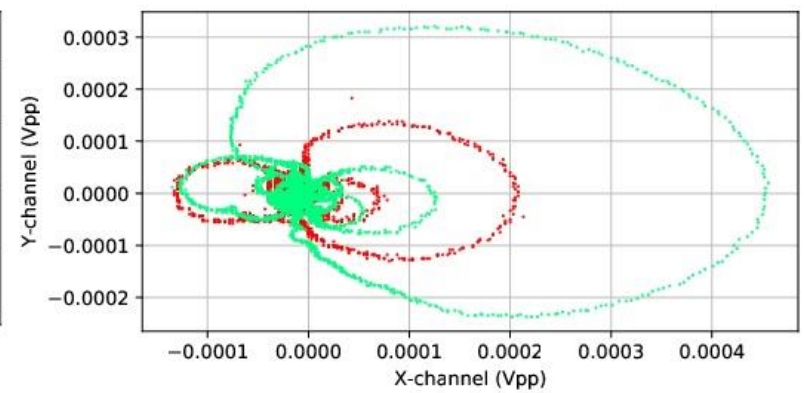

(d)

Figure 12. Zoomed in version of Figure 11. Three peaks can be identified at about 27160, 37430, and $45940 \mathrm{~Hz}$. (a) The $\mathrm{x}$-channel is the quadrature signal. (b) The y-channel is the in-phase signal. (c) The r-channel is the amplitude of the signal. (d) The Johnson-Nyquist plot.

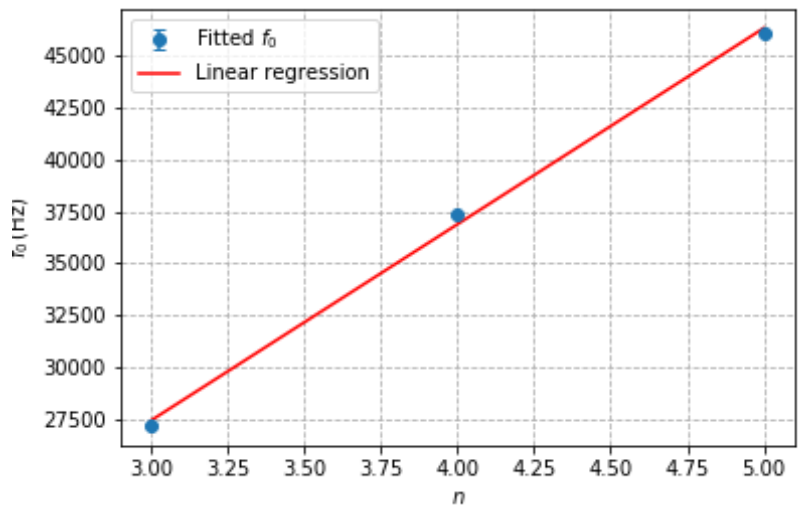

Figure 13. $\mathrm{f}_{0}$ vs. $\mathrm{n}$ plot. The dots are $\mathrm{f}_{0}$ obtained from the Lorentzian fits on the data obtained in ${ }^{4} \mathrm{He}$ gas at $1.2 \times 1 \mathbf{1 0}^{5}$ $\mathrm{Pa}$, room temperature. The line is generated by linear regression.

Again, a linear fit can be performed on the $f_{0}$ 's by assigning an integer to each one of them ( 3,4 , and 5 are used in this case). The fitting line for $\mathrm{P}=1.2 \times 10^{5} \mathrm{~Pa}$ is shown in Fig. 13. The fitting line for the speed of sound at $\mathrm{P}=1.6 \times 10^{5} \mathrm{~Pa}$ is near identical to $\mathrm{P}=1.2 \times 10^{5}$.

For Figure 13, the fitted slope is $\Lambda=9448.94 \pm 0.94 \mathrm{~Hz}$. The intercept equals to $-913.94 \pm 4.16 \mathrm{~Hz}$. Following the same process, it is calculated that $c=1036.81 \mathrm{~m} / \mathrm{s}$. 
Similarly for $\mathrm{P}=1.6 \times 10^{5} \mathrm{~Pa}$, the calculation for $\Lambda=9391.07 \pm 0.86 \mathrm{~Hz}$, and an intercept of $-721.11 \pm 4.06 \mathrm{~Hz}$. Calculations give $\mathrm{c}=1030.46 \mathrm{~m} / \mathrm{s}$. For $\mathrm{N}_{2}$ at $\mathrm{P}=1.0 \times 10^{5} \mathrm{~Pa}$, the calculation for $\Lambda=3116.45 \pm 0.07 \mathrm{~Hz}$, and an intercept of $727.26 \pm 0.61 \mathrm{~Hz}$. Calculations give $\mathrm{c}=341.96 \mathrm{~m} / \mathrm{s}$.

The calculations were compared to literature values (McCarty, 1973), showing a percentage error ranging from $1.03 \%$ to $2.55 \%$, using the equation

$$
\text { Percentage error }=\left|\frac{\mathrm{c}_{\mathrm{e}}-c_{l}}{c_{l}}\right| \times 100 \% \text {; }
$$

where $\mathrm{c}_{1}$ and $c_{e}$ are the literature and experimental speeds of sound, respectively.

\section{Conclusion}

We constructed and tested the SHeEP project as an alternate system to purify ${ }^{3} \mathrm{He}$ gas. Knowing the length of the cavity and fitted slope of the harmonic frequencies vs. $\mathrm{n}$ allow the speed of sound to be determined. The speed of sound was measured for various samples at room temperature and different pressures. Further work is required to test the accuracy of the acoustic cavity at determining the concentration level for a binary gas mixture.

\section{Acknowledgements}

I wish to first express my sincere thanks to Dr. Yoonseok Lee, for providing me with all the necessary facilities for the research. Wenguang Jiang for providing patience, wisdom, encouragement, guidence and friendship throughout the years of this research. Finally but not least, my family for supplying an unsurrendering fire within me and for understanding for the moments I was not physically or mentally present in their lives.

\section{References}

Blundell, S.J., Blundell, K. M. (2010). Concepts in Thermal Physics, Second Edition. New York, NY: Oxford University Press Inc.

Dobbs, E. R. (1994). Helium Three, First Edition. New York, NY: Oxford University Press Inc.

Dmitriev, V. V., Zavjalov, V. V., Zmeev, D. Y., \& Levitin, L. V. (2004). Purification of ${ }^{3} \mathrm{He}$ from a ${ }^{4} \mathrm{He}$ impurity using adsorption. Instruments and Experimental Techniques, 47(4), 567-569. https://doi.org/10.1023/B:INET.0000038411.46876.ed.

Feder, Toni (2009). US government agencies work to minimize damage due to helium-3 shortfall. Physics Today 62, 10, 21; doi:10.1063/1.3248465

Kagiwada, R. S., and Rudnick, I. (1970). Note on a simple method for determining the isotopic concentration of a ${ }^{3} \mathrm{He}-{ }^{4} \mathrm{He}$ gas. Journal of Low Temperature Physics, 3(1), 113-114. https://doi.org/10.1007/BF00628401. 
Kinsler, L. E., Frey, A. R., Coppens, A. B., Sanders, J. V. (1999). Fundamentals of Acoustics, Fourth Edition. Hoboken, NJ: John Wiley \& Sons, Inc.

Lin, H., Feng, X. J., Gillis, K. A., Moldover, M. R., Zhang, M. R., Sun J. P. and Duan, Y. Y. (2013). Improved determination of the Boltzmann constant using a single fixed length cylindrical cavity. Metrologia, 50(5), 417-432. https://doi.org/10.1088/0026-1394/50/5/41.

McCarty, R. D. (1973). Thermodynamics properties of Helium 4 from 2 to $1500 \mathrm{~K}$ at pressures to $10^{8} \mathrm{~Pa}$. Journal of Physical and Chemical Reference Data, 2, 923. https://doi.org/10.1063/1.3253133.

Polturak, E., Garrett, S. L., Lipson, S. G. (1986). Precision acoustic gas analyzer for binary mixtures. Review of Scientific Instruments 57, 2837. http://dx.doi.org/10.1063/1.1139053. 